Pacific Journal of Mathematics

OPERATOR ALGEBRAS WITH REDUCING INVARIAN 


\title{
OPERATOR ALGEBRAS WITH REDUCING INVARIANT SUBSPACES
}

\author{
T. B. HoOver
}

\begin{abstract}
A weakly closed algebra of operators on a Hilbert space is reductive if every subspace which is invariant for the algebra reduces. If $\mathscr{A}$ is a reductive algebra, let $\mathscr{F}$ be the von Neumann algebra genenerated by the projections which commute with $\mathscr{A}$. If $\mathscr{F}$ is properly infinite, or it $\mathscr{F}$ has a cyclic vector, then $\mathscr{A}$ is self-adjoint. If $\mathscr{F}$ has no direct summand which is abelian and of infinite uniform multiplicity, then $\mathscr{F}$ is the commutant of $\mathscr{A}$.
\end{abstract}

I. Introduction. In what follows, $\mathscr{H}$ denotes a Hilbert space (all our Hilbert spaces are complex). The term operator is used to refer to a bounded linear transformation, usually on $\mathscr{H}$, and operator algebra or simply algebra means a weakly closed algebra of operators which contains the identity operator. The algebra of all operators on $\mathscr{H}$ is denoted by $\mathscr{L}(\mathscr{H})$, and if $\mathscr{S}$ is a subset of $\mathscr{L}(\mathscr{H}), \mathscr{S}^{\prime}$ is the algebra of all operators which commute with $\mathscr{S}$. Finally, an algebra $\mathscr{A}$ on $\mathscr{C}$ is called reductive if every invariant subspace is reducing. The study of reductive algebras was initiated by Radjavi and Rosenthal in [10] where they extend to reductive algebras many of the known theorems about transitive algebras (algebras with no proper invariant subspaces). Certainly every self-adjoint algebra is reductive, and, as Radjavi and Rosenthal point out, the major question in the theory of reductive algebras is, are there any non-selfadjoint reductive algebras? A negative to this question would solve all the well known invariant subspace questions. In $\S 2$ of this paper we study a reductive algebra $\mathscr{A}$ by borrowing conditions from the theory of von Nemann algebras to impose on the lattice of projections in $\mathscr{A}^{\prime}$.

Following Dyer, Pederson and Porcelli [7], we call an operator reductive if the algebra it generates is reductive. In $\S 3$ we use the results of $\S 2$ to show that every reductive operator is the direct sum of a normal operator and a reductive operator of a special kind.

II. The invariant algebra. Let $\mathscr{A}$ be a reductive algebra. The invariant algebra for $\mathscr{A}$, writted $\mathscr{I}(\mathscr{A})$ or simply $\mathscr{I}$, is defined to be the algebra generated by the projections $P_{\mathscr{N}}$ where $\mathscr{C}$ ranges over all the invariant subspaces of $\mathscr{A}$, and $P_{\mathscr{M}}$ denotes the orthogonal projection onto $\mathscr{K}$. Since the $P_{\mathscr{M}}$ are self-adjoint, $\mathscr{I}$ is 
a von Neumann (self-adjoint) algebra. Further, each $\mathscr{l}$ reduces $\mathscr{A}$, so the $P_{\mathscr{A}}$ are in $\mathscr{A}^{\prime}$ and thus $\mathscr{J}$ is a subalgebra of $\mathscr{A}^{\prime}$. In fact, $\mathscr{F}$ is the largest self-adjoint sub-algebra of $\mathscr{A}^{\prime}$. For any algebra it is true that $\mathscr{A}$ is in $\mathscr{A}^{\prime \prime}$. But since our $\mathscr{A}$ is reductive, $\mathscr{J}$ is in $\mathscr{A}^{\prime}$ or $\mathscr{I}^{\prime}$ contains $\mathscr{A}^{\prime \prime}$. Therefore $\mathscr{A} \cong \mathscr{I}^{\prime}$, and $\mathscr{I}^{\prime}$ is the smallest self-adjoint algebra with this property. Thus we must discover when $\mathscr{A}=\mathscr{I}^{\prime}$.

The following corollary to a theorem of Douglas, Pearcy, Herrero and Salinas [5], [8] will be useful throughout this paper.

CoRollary 1. If $\mathscr{A}$ is a reductive algebra and if $P$ is a projection in the center of $\mathscr{F}$, then $P$ is in $\mathscr{A}^{\prime \prime}=\left(\mathscr{A}^{\prime}\right)^{\prime}$.

Proof. If $Q$ is the projection onto any invariant subspace of $\mathscr{A}$, then since $P$ and $Q$ commute, $\|P-Q\|=1$. It follows by [8, Corollary 4.4] that the range of $P$ is invariant for $\mathscr{A}^{\prime}$. But the same is true of $1-P$, so $P(\mathscr{H})$ reduces $\mathscr{A}^{\prime}$ and $P$ is in $\mathscr{A}^{\prime \prime}$.

If $\mathscr{A}$ is any algebra and if $P$ is a projection in $\mathscr{A}^{\prime}$, then $\mathscr{A}_{P}$ denotes the restriction of $\mathscr{A}$ to the range of $P$. We call $\mathscr{A}_{P}$ a part of $\mathscr{A}$. If $\mathscr{A}$ is reductive, then the weak closure of $\mathscr{A}_{P}$ is too, and if $P$ is central in $\mathscr{I}(\mathscr{A})$, then $\left.(\mathscr{I}(\mathscr{A}))_{P}=\mathscr{I}(\mathscr{A})_{P}\right)$. Our plan is to break up the algebra $\mathscr{I}$ into parts of pure type and watch what happens to the corrosponding parts of $\mathscr{A}$. Recall that if $\alpha$ is one of the symbols $I I_{1}, I I_{\infty}, I I I$ or $I_{n}$ with $1 \leqq n \leqq \operatorname{dim}(\mathscr{C})$, then there is a possibly zero central projection $P_{\alpha}$ in $\mathscr{I}$ such that $\mathscr{I}_{P_{\alpha}}$ is of type $\alpha$. Further, these projections are pairwise orthogonal and sum to 1 . The pertinent definitions and theorems may be found in [9].

Before we can prove our first theorem we need some more notation. If $n$ is a cardinal number, $\mathscr{P}^{(n)}$ denotes the orthogonal direct sum of $n$ copies of the Hilbert space $\mathscr{H}$, and for $A$ in $\mathscr{L}(\mathscr{H}), A^{(n)}$ denotes the operator on $\mathscr{H}^{(n)}$ which is a direct sum of $n$ copies of $A$. For an algebra $\mathscr{A}$ on $\mathscr{H}, \mathscr{A}^{(n)}$ is the algebra on $\mathscr{H}^{(n)}$ consisting of all $A^{(n)}$ with $A$ in $\mathscr{A}$. Finally, $M_{n}(\mathscr{A})$ is used to denote the algebra of $n \times n$ matrices with entries in $\mathscr{A}$ where the elements of $M_{n}(\mathscr{A})$ act on $\mathscr{C}^{(n)}$ in the usual fashion. (If $n$ is infinite, then $M_{n}(\mathscr{A})$ consists only of those matrices which represent bounded

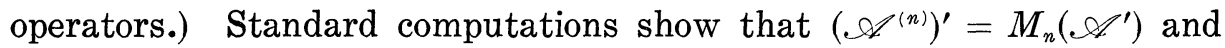
$\left(M_{n}(\mathscr{A})\right)^{\prime}=\mathscr{A}^{\prime(n)}$.

THEOREM 2. If $\mathscr{A}$ is a reductive algebra and if its invariant algebra $\mathscr{I}$ is properly infinite $\left(P_{\alpha}=0\right.$ if $\alpha=I I_{1}$ or $I_{n}$ with $\left.n<\infty\right)$ then $\mathscr{A}$ is self-adjoint. 
Proof. By [10, Lemma 2], it suffices to show that $\mathscr{A}^{(n)}$ is reductive every finite $n$. According to $[4, \mathrm{p} .298$, Corollary 2] there is a sequence $\left\{P_{i}\right\}$ of pairwise orthogonal projections in $\mathscr{J}$ such that $\Sigma P_{i}=I$ and $P_{i}$ is equivalent to $I$ in the sense of von Neumann $\left(P_{i} \sim I\right)$. A routine argument now shows that $\mathscr{A}$, as a subalgebra of $\mathscr{I}^{\prime}$, is unitarily equivalent to $\mathscr{N}^{N_{0}}$. From this it follows that for every finite $n, \mathscr{A}$ is unitarily equivalent to $\mathscr{A}^{(n)}$. The property of being reductive is preserved under unitary equivalence, so $\mathscr{L}^{(n)}$ is reductive for every finite $n$ and theorem is proved.

For our next theorem, we need more terminology. If $\mathscr{B}$ is a maximal abelian self-adjoint algebra, then we say the algebra $\mathscr{B}^{(n)}$ has uniform multiplicity $n$. It is well known that any abelian von Neumann algebra $\mathscr{B}$ can be written as an orthogonal direct sum $\mathscr{B}=\Sigma \oplus \mathscr{B}_{n}$ where $n$ ranges over a subset of the set of cardinal numbers between 1 and the dimension of $\boldsymbol{\aleph}$, and $\mathscr{B}_{n}$ is of uniform multiplicity $n$.

Observe that if an algebra $\mathscr{A}$ is not only reductive but selfadjoint, then $\mathscr{F}=\mathscr{A}^{\prime}$.

THEOREM 3. If $\mathscr{A}$ is a reductive algebra, and if its invariant algebra $\mathscr{I}$ contains no central projection $P$ such that $\mathscr{I}_{P}$ is abelian and of infinite uniform multiplicity, then $\mathscr{A}^{\prime}=\mathscr{F}$.

Proof. By Corollary 1, if $P$ is a projection in the center of $\mathscr{J}$, then $P$ is also in the center of $\mathscr{A}^{\prime}$. It follows that

$$
\begin{aligned}
\mathscr{A}^{\prime} & =\mathscr{A}_{P}^{\prime} \oplus \mathscr{A}_{1-P}^{\prime}=\left(\mathscr{A}_{P}\right)^{\prime} \oplus\left(\mathscr{\mathscr { A }}_{1-P}\right)^{\prime} \\
& =\left(\operatorname{cl}\left(\mathscr{A}_{P}\right)\right)^{\prime} \oplus\left(\mathrm{cl}\left(\mathscr{A}_{1-P}\right)\right)^{\prime}
\end{aligned}
$$

where cl denotes the weak closure. Thus it suffices to consider the special cases in which $\mathscr{F}$ is of pure type. If $\mathscr{F}$ is type $I I I, I I_{\infty}$, or $I_{n}$ with $n$ infinite, then by Theorem $2, \mathscr{A}$ is self-adjoint and $\mathscr{A}^{\prime}=\mathscr{J}$.

If $\mathscr{F}$ is of type $I I_{1}$, then by [9, Theorem 49], there are orthogonal projections $P_{1}$ and $P_{2}$ in $\mathscr{F}$ such that $P_{1} \sim P_{2}$ and $P_{1}+P_{2}=1$. Thus $\mathscr{A}$ is unitarily equivalent to the algebra $\mathscr{B}^{(2)}$ where $\mathscr{B}=\mathscr{A}_{P_{1}}$, and $\mathscr{A}^{\prime}$ is $M_{2}\left(\mathscr{B}^{\prime}\right)$. We know that $\mathscr{J}$ is the largest self-adjoint subalgebra of $\mathscr{L}^{\prime}$. Therefore to complete this case, it suffices to show that $\mathscr{A}^{\prime}$ is self-adjoint but this will be true if and only if $\mathscr{B}^{\prime}$ is self-adjoint. Suppose $T$ is an operator on $P_{1}(\mathscr{C})=\mathscr{K}$ which commutes with $\mathscr{B}$. The subspace $\mathscr{C}$ defined by

$$
\mathscr{C l}=\left\{\left(x_{1} T x\right): x \in \mathscr{K}\right\}
$$


is invariant for $\mathscr{B}^{(2)}$ and, since $\mathscr{B}^{(2)}$ is reductive $\mathscr{C l}$ reduces $\mathscr{B}^{(2)}$. That is, for every $B$ in $\mathscr{B}$ and $x$ in $\mathscr{K},\left(B^{*}\right)^{(2)}(x, T x)=\left(B^{*} x, B^{*} T x\right) \epsilon$ IC. This can happen if and only if $T B^{*} x=B^{*} T x$. But $x$ is arbitrary in $\mathscr{H}$, so for every $B$ in $\mathscr{B}, T$ commutes with $B^{*}$. Thus $T^{*}$ is in $\mathscr{B}^{\prime}$ and this case is complete.

If $\mathscr{F}$ is of type $I_{n}, 2 \leqq n<\infty$, we can use the same argument given above, only we need $n$ equivalent projections instead of just 2 .

There remains the case in which $\mathscr{F}$ is type $I_{1}$, that is, $\mathscr{F}$ is abelian. Here again it suffices to look at special cases separatly. Therefore we assume $\mathscr{J}$ is of uniform multiplicity $n$ with $n$ finite; say $\mathscr{F}=\mathscr{B}^{(n)}$ where $\mathscr{B}$ is a maximal abelian self-adjoint algebra on a Hilbert space $\mathscr{K}$, and $\mathscr{H}=\mathscr{K}^{(n)}$. Suppose $T$ is an operator which commutes with $\mathscr{A}$. By Corollary 1 , and the fact that $\mathscr{J}$ is abelian, $T$ commutes with the projections in $\mathscr{F}$ and so $T$ is in $\mathscr{J}^{\prime}$. If $n=1$, so that $\mathscr{F}$ is maximal abelian, then $\mathscr{F}=\mathscr{J}^{\prime}$ and we are done. If $n>1$, then $\mathscr{J}^{\prime}=M_{n}(\mathscr{B})$ and in particular $T=\left(T_{i j}\right)_{i, j=1}^{n}$ with each $T_{i j}$ in $\mathscr{B}$. By [3, Theorem 2], there is a unitary operator $U$ in $\mathscr{I}^{\prime}$ such that $U T U^{*}$ is in upper triangular form; that is, $U T U^{*}=\left(S_{i j}\right)_{i, j=1}^{n}$ with $S_{i j}$ in $\mathscr{B}$ and $S_{i j}=0$ for $i>j$. The algebra $U \mathscr{A} U^{*}=\left\{U A U^{*}, A \in \mathscr{A}\right\}$ is also reductive with invariant algebra $U \mathscr{J} U^{*}=\mathscr{J}$. Therefore, by replacing $\mathscr{A}$ by $U \mathscr{A} U^{*}$, we may assume that $T$ itself is upper triangular.

Let $T^{\prime}=T-T_{11}^{(n)} . T^{\prime}$ is in $\mathscr{A}^{\prime}$ since both $T$ and $T_{11}^{(n)}$ are. It follows that $\mathscr{C}$, the kernel of $T^{\prime}$ is invariant for $\mathscr{A}$. But $T^{\prime}$ has all zeros in the first column, so $\mathscr{C}$ contains all vectors of the form $(x, 0, \cdots, 0), x$ in $\mathscr{K}$. The only projection in $\mathscr{F}$ which leaves such vectors fixed is 1. Therefore $\mathscr{M}=\mathscr{H}, T^{\prime}=0$ and $T=T_{11}^{(n)}$ which is in $\mathscr{J}$.

A set $S$ in $\mathscr{H}$ is cyclic for $\mathscr{F}$ if the $\operatorname{set}\{B x: B \in \mathscr{J}, x \in S\}$ is dense in $\mathscr{H}$, and a vector $x$ is cyclic if $\{x\}$ is a cyclic set. The following theorem and its proof are closely related to a theorem of Sarason [12].

THEOREM 4. If $\mathscr{A}$ is a reductive algebra whose invariant algebra $\mathscr{F}$ has a cyclic vector, then $\mathscr{A}$ is self-adjoint.

Proof. Let $A$ be any operator in $\mathscr{A}$ and let

$$
U=\left\{T:\left\|\left(T-A^{*}\right) x_{i}\right\|<\varepsilon i=1 \cdots n\right\}
$$

be any basic strong neighborhood of $A^{*}$. The function $\phi$ which maps $T$ to $\sum_{i=1}^{n}\left(T x_{i}, x_{i}\right)$ is a positive, normal linear functional on $\mathscr{J}^{\prime}$. But 
has a cyclic vector, so $\mathscr{F}^{\prime}$ has a separating vector, and by [4, p. 222, Theorem 4] there is a vector $x_{0}$ in $\mathscr{H}$ such that $\phi(T)=\left(T x_{0}, x_{0}\right)$ for each $T$ in $\mathscr{I}^{\prime}$. Let $\mathscr{C}$ be the smallest subspace of $\mathscr{H}$ which is invariant for $\mathscr{A}$ and which contains $x_{0}$. The subspace $\mathscr{C}$ reduces $\mathscr{A}$, so that $A^{*}(\mathscr{C}) \subset \mathscr{C}$ and in particular, $A^{*} x_{0}$ is in $\mathscr{C l}$. Thus there is a $B$ in $\mathscr{A}$ such that $\left\|B x_{0}-A^{*} x_{0}\right\|<\varepsilon$. On the other hand

$$
\begin{aligned}
\varepsilon^{2}>\left\|B x_{0}-A^{*} x_{0}\right\| & =\left(\left(B-A^{*}\right)^{*}\left(B-A^{*}\right) x_{0}, x_{0}\right) \\
& =\sum_{i=1}^{n}\left(\left(B-A^{*}\right)^{*}(B-A) x_{i}, x_{i}\right) \\
& =\sum_{i=1}^{n}\left\|B-A^{*} x_{i}\right\|^{2} .
\end{aligned}
$$

Therefore $\left\|\left(B-A^{*}\right) x_{i}\right\|<\varepsilon$ for each $i, B$ is in $U$, and the theorem is proved.

COROLlaRy 5. If the invariant algebra $\mathscr{F}$ of a reductive algebra $\mathscr{A}$ has a cyclic set $S$ of cardinality $n<\infty$, and if $\mathscr{A}^{(n)}$ is also reductive, then $\mathscr{A}$ is self-adjoint.

Proof. $\mathscr{A}^{(n)}$ has invariant algebra $M_{n}(\mathscr{J})$, and if $S=\left\{x_{1}, \cdots, x_{n}\right\}$, then the vector $\left(x_{1}, \cdots, x_{n}\right)$ is easily seen to be cyclic for $M_{n}(\mathscr{J})$. By Theorem 4, $\mathscr{A}^{(n)}$ is self-adjoint and so is $\mathscr{A}$.

It should be noted that Corollary 5 is still true if $n$ is infinite and the existance of the cyclic set $S$ is not necessary. In this situation, $\mathscr{I}\left(\mathscr{A}^{(n)}\right)$ is properly infinite so by Theorem $2 \mathscr{A}^{(n)}$ is self-adjoint and so is $\mathscr{A}$.

III. Reductive operators. In this section we are interested in reductive operators-operators which generate reductive algebras. If $A$ is a reductive operator and $\mathscr{A}$ is the algebra generated by $A$, then we write $\mathscr{J}(A)$ for $\mathscr{J}(\mathscr{A})$. Porcelli, Pederson and Dyer [7] have shown that every reductive operator on a separable Hilbert space is normal if and only if every operator has a proper invariant subspace. Ando [1] has proved that compact reductive operators are normal, and Rosenthal [11] has proved the same result for polynomially compact operators.

THEOREM 6. If $A$ is a reductive operator on a Hilbert space $\mathscr{H}$, then $A$ can be written as a direct sum $A_{1} \oplus A_{2}$ where $A_{1}$ is normal $A_{2}$ is reductive and $\mathscr{F}\left(A_{2}\right)$ is abelian and of uniform multiplicity $\aleph_{0}$. Furthermore, $\{A\}^{\prime}=\left\{A_{1}\right\}^{\prime} \oplus\left\{A_{2}\right\}^{\prime}$ and all the invariant subspaces of $A_{2}$ are hypervariant (invariant for $\left.\left\{A_{2}\right\}^{\prime}\right)$. 
Proof. Let $P_{i} i=1,2,3$ be the projections in the center of $\mathscr{J}=\mathscr{J}(A)$ such that $\mathscr{J}_{P_{1}}$ has no central projection $Q$ for which $\left(\mathscr{I}_{P_{1}}\right)_{Q}=\mathscr{I}_{Q}$ is abelian and of infinite uniform multiplicity, $\mathscr{I}_{P_{2}}$ is abelian and of uniform multiplicity $\boldsymbol{\aleph}_{0}$, and $\mathscr{I}_{P_{3}}$ is abelian and has no direct summand of countable uniform multiplicity. We let $A_{i}$ be restriction of $A$ to the range of $P_{i}$. Clearly $A=A_{1} \oplus A_{2} \oplus A_{3}$, each $A_{i}$ is reductive, and the invariant algebra $\mathscr{J}\left(A_{i}\right)$ is $\mathscr{F}_{P_{i}}$. All the projections in $\mathscr{F}_{P_{3}}$ have nonseparable ranges, so $A_{3}$ has no nonzero separable invariant subspaces. On the other hand if $x$ is a nonzero vector in the range $P_{3}$, then the smallest subspace which contains $x$ and which is invariant for $A_{3}$ is separable. This is a contradiction unless the direct summand $A_{3}$ is missing. Certainly $A_{1}$ is in $\left\{A_{1}\right\}^{\prime}$, but by Theorem $3,\left\{A_{1}\right\}^{\prime}$ is the self-adjoint algebra $\mathscr{F}\left(A_{1}\right)$, so that $A_{1}^{*}$ is in $\left\{A_{1}\right\}^{\prime}$ and $A_{1}$ is normal. Corollary 1 tells us that the projections in $\mathscr{F}_{A_{3}}$ are also in $\left\{A_{2}\right\}^{\prime \prime}$, so that all the invariant subspaces of $A_{2}$ are invariant for $\left\{A_{2}\right\}^{\prime}$. Finally, $P_{1}$ is in the center of $\{A\}^{\prime}$, so $\{A\}^{\prime}=\left\{A_{1}\right\}^{\prime} \oplus\left\{A_{2}\right\}^{\prime}$.

The following corollary is a consequence of the first few lines of the proof of Theorem 6 .

COROLlARY 7. If $\mathscr{A}$ is a reductive algebra whose invariant algebra has no abelian part of infinite multiplicity, then every operator in the center of $\mathscr{A}$ is normal.

Corollary 8. Let $A=A_{1} \oplus A_{2}$ be a reductive operator written according to its decomposition given in Theorem 6. A subspace $\mathscr{H}$ is hyperinvariant for $A$ if and only if $\mathscr{H}=\mathscr{C}_{1} \oplus \mathscr{L}_{2}$ where $\mathscr{M}_{1}$ is the range of a spectral projection for $A_{1}$ and $\mathscr{L}_{2}$ is invariant for $A_{2}$.

Proof. Since $\{A\}^{\prime}=\left\{A_{1}\right\} \oplus\left\{A_{2}\right\}$, a subspace $\mathscr{C l}$ will be hyperinvariant for $A$ just in case it is the form $\mathscr{L}_{1} \oplus \mathscr{C}_{2}$ where $\mathscr{A}_{i}$ is hyperinvariant for $A_{i}$. We know that all the invariant subspaces of $A_{2}$ are hyperinvariant, while the normal operator $A_{1}$ has the ranges of its spectral projections for its hyperinvariant subspaces [5].

CoRollary 9. A nonscalar reductive operator $A$ which has an invariant subspace, has a hyperinvariant subspace.

Proof. Write $A$ as $A_{1} \oplus A_{2}$ according to Theorem 6. The operators $A_{1}$ and $A_{2}$ satisfy the conclusion of this corollary so $A$ must also.

IV. Remarks. A special kind of reductive algebra which is of particular interest in the transitive algebra-an algebra with no proper 
invariant subspaces [2].

If $\mathscr{A}$ is a transitive algebra on an infinite dimensional Hilbert space, then the invariant algebra $\mathscr{F}(\mathscr{A})$ is the algebra of all multiples of the identity, which is an abelian algebra of uniform multiplicity equal to the dimension of $\mathscr{H}$. Even to show that the commutant of a transitive algebra is the invariant algebra would solve the hyperinvariant subspace question [6]. Therefore it is not surprising that we have learned nothing about reductive algebras with abelian invariant algebras of infinite multiplicity.

There are, however, other special reductive algebras which offer hope. Must a reductive algebra with an abelian invariant algebra of finite uniform multiplicity be self-adjoint? What if the invariant algebra is type $I I_{1}$ ? The case in which the invariant algebra is type $I_{n}, n$ finite, is very close to the abelian case, and one would expect the difficulties from the latter to carry over to the former.

\section{REFERENCES}

1. T. Ando, A note on invariant subspaces of a compact normal operator, Arch. Math., 14 (1963), 337-340.

2. W. B. Arveson, A density theorem for operator algebras, Duke Math. J., 34 (1967), 635-647.

3. D. Deckard and C. Pearcy, On matrices over the ring of continuous complex-valued functions on a Stonian space, Proc. Amer. Math. Soc., 14 (1963), 322-328.

4. J. Dixmier, Les algebres d'opérateurs dans l'espace Hilbertian, 2nd edition, GauthierVillars, Paris, 1969.

5. R. G. Douglas and C. Pearcy, On a topology for invariant subspaces, J. Funct. Anal., 2 (1968), 323-341.

6. — Hyperinvariant subspaces and transitive algebras, Michigan Math. J., (to appear).

7. J. Dyer, E. A. Pederson, and P. Porcelli, An equivalent formulation of the invariant subspace conjecture, Ball. Amer. Math. Soc., (to appear).

8. D. Herrero and N. Salinas, Analytically invariant and bi-invariant subspaces, (to appear).

9. I. Kaplansky, Rings of Operators, W. A. Benjamin, Inc., New York, 1968.

10. H. Radjavi and P. Rosenthal, A sufficient condition that an operator algebra be self-adjoint, (to appear)

11. P. Rosenthal, Completely reducible operators, Proc. Amer. Math. Soc., 19 (1968), 826-830.

12. D. Sarason, Invariant subspaces and unstarred operator algebras, Pacific J. Math., 17 (1966), 511-517.

Received September 29, 1971.

LoUisiana State UNIVERSITY 



\section{PACIFIC JOURNAL OF MATHEMATICS}

\section{EDITORS}

H. SAMELSON

Stanford University

Stanford, California 94305

C. R. Hовву

University of Washington

Seattle, Washington 98105
J. DugundJI

Department of Mathematics University of Southern California

Los Angeles, California 90007

RICHARD ARENS

University of California

Los Angeles, California 90024

\section{ASSOCIATE EDITORS}

E. F. BECKENBACH

B. H. NeumanN

F. WOLF

K. YoSHIDA

\section{SUPPORTING INSTITUTIONS}

UNIVERSITY OF BRITISH COLUMBIA

CALIFORNIA INSTITUTE OF TECHNOLOGY

UNIVERSITY OF CALIFORNIA

MONTANA STATE UNIVERSITY

UNIVERSITY OF NEVADA

NEW MEXICO STATE UNIVERSITY

OREGON STATE UNIVERSITY

UNIVERSITY OF OREGON

OSAKA UNIVERSITY
UNIVERSITY OF SOUTHERN CALIFORNIA

STANFORD UNIVERSITY

UNIVERSITY OF TOKYO

UNIVERSITY OF UTAH

WASHINGTON STATE UNIVERSITY

UNIVERSITY OF WASHINGTON

AMERICAN MATHEMATICAL SOCIETY

NAVAL WEAPONS CENTER

Printed in Japan by International Academic Printing Co., Ltd., Tokyo, Japan 


\section{Pacific Journal of Mathematics}

\section{Vol. 44, No. $1 \quad$ May, 1973}

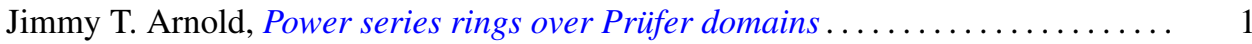

Maynard G. Arsove, On the behavior of Pincherle basis functions . . . . . . . . . 13

Jan William Auer, Fiber integration in smooth bundles ................. 33

George Bachman, Edward Beckenstein and Lawrence Narici, Function algebras

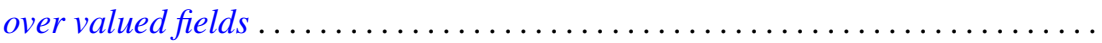

Gerald A. Beer, The index of convexity and the visibility function . . . . . . . . . . .

James Robert Boone, A note on mesocompact and sequentially mesocompact

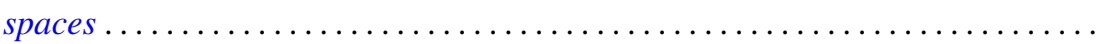

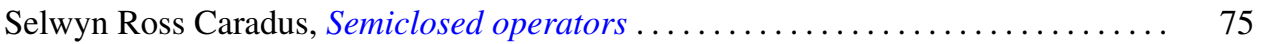

John H. E. Cohn, Two primary factor inequalities . . . . . . . . . . . . . . . 81

Mani Gagrat and Somashekhar Amrith Naimpally, Proximity approach to

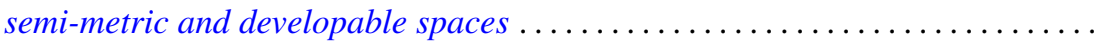

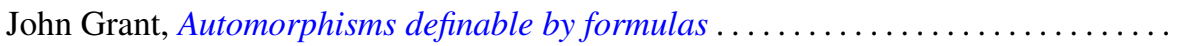

Walter Kurt Hayman, Differential inequalities and local valency ..............

Wolfgang H. Heil, Testing 3-manifolds for projective planes . . . . . . . . . . . . .

107

Melvin Hochster and Louis Jackson Ratliff, Jr., Five theorems on Macaulay

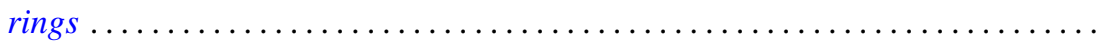

Thomas Benton Hoover, Operator algebras with reducing invariant subspaces ....

James Edgar Keesling, Topological groups whose underlying spaces are separable

Fréchet manifolds...

Frank Leroy Knowles, Idempotents in the boundary of a Lie group . .

191

George Edward Lang, The evaluation map and EHP sequences ...

201

Everette Lee May, Jr, Localizing the spectrum . . . . . . . . . . . .

211

Frank Belsley Miles, Existence of special $K$-sets in certain locally compact abelian groups.

Susan Montgomery, A generalization of a theorem of Jacobson. II . .

T. S. Motzkin and J. L. Walsh, Equilibrium of inverse-distance forces in

three-dimensions.

Arunava Mukherjea and Nicolas A. Tserpes, Invariant measures and the converse

of Haar's theorem on semitopological semigroups .

James Waring Noonan, On close-to-convex functions of order $\beta$

Donald Steven Passman, The Jacobian of a growth transformation

Dean Blackburn Priest, A mean Stieltjes type integral ........ .

Joe Bill Rhodes, Decomposition of semilattices with applications to topological

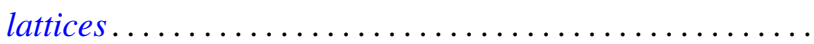

Claus M. Ringel, Socle conditions for $\mathrm{QF}-1$ rings ..........

Richard Rochberg, Linear maps of the disk algebra

Roy W. Ryden, Groups of arithmetic functions under Dirichlet convolution . .

Michael J. Sharpe, A class of operators on excessive functions

Erling Stormer, Automorphisms and equivalence in von Neumann algebras ..

Philip C. Tonne, Matrix representations for linear transformations on series

analytic in the unit disc. 